\title{
Évolution de l'industrie mondiale de l'aluminium sur une longue période : une approche par les prix
}

\author{
Jasmin Tremblay
}

Quelles tendances de long terme montrent les principaux indicateurs de l'industrie mondiale de l'aluminium ? Comment ceux-ci évolueront-ils dans les vingt prochaines années? Il va sans dire que la prévision économique est un art difficile. Gilbert ${ }^{1}$ suggère que, même en utilisant des techniques statistiques avancées, au-delà d'un horizon d'un à deux ans, on ne peut pas espérer faire de meilleures prévisions qu'avec des modèles plus intuitifs. Cela pourrait être dû au fait que le marché lui-même ne possède pas l'information pertinente aux prévisions de long terme. Cela étant dit, après avoir donné un aperçu global de l'industrie de l'aluminium, nous indiquerons les tendances qui ont marqué l'industrie mondiale de l'aluminium dans les dernières années, puis nous spéculerons sur son évolution future.

Pour les fins de notre analyse économique, il faut définir plus précisément le marché dont il

\section{Tendances des vingt dernières années}

Une façon de résumer l'activité économique sur un marché est d'y observer l'évolution des prix. La volonté d'expliquer cette évolution nous pousse ensuite à examiner d'autres variables et on peut ainsi avoir un portrait relativement complet et cohérent de la situation. Lorsqu'on considère l'évolution à long terme d'une variable économique comme un prix, il faut pouvoir tenir compte du fait que l'ensemble des prix connaît un mouvement général à la hausse, c'est-à-dire qu'il y a de l'inflation. C'est pourquoi, en analyse économique, on utilise un prix dit «réel », qui est obtenu en pondérant le prix courant d'un sera question. La figure 1 donne une représentation schématique des produits (ellipses) et des procédés (rectangles) relatifs à l'aluminium. Chaque produit, ou certaines combinaisons ou décompositions de produits dans ce schéma, peut être pris à part en tant qu'objet d'un marché. Ici, notre attention se fixera sur l'aluminium primaire. Le schéma nous indique donc que sur ce marché, l'alumine est une matière première, les offreurs sont les entreprises qui produisent de l'aluminium primaire, les demandeurs sont les entreprises qui en achètent pour en faire la première transformation et l'aluminium secondaire est considéré comme un des substituts de l'aluminium primaire, c'est-à-dire comme un bien qui peut remplacer l'aluminium primaire dans certains de ses usages.

bien particulier à l'aide d'un indice de prix approprié, qui contient de l'information sur l'évolution du prix moyen d'un ensemble beaucoup plus large de biens. On peut supposer que le prix courant est souvent utilisé dans les décisions de gestion, y compris lorsqu'on fait des prévisions sur un horizon de plusieurs années. Or, prendre une décision d'expansion de la capacité de production, par exemple, parce qu'on prévoit que le prix courant du bien produit va augmenter, c'est un peu comme se dire, en participant à une course, que tout va bien parce qu'on a réussi à avancer, 
alors qu'il faut aussi se préoccuper de la position des autres coureurs.

Comment le prix de l'aluminium est-il déterminé ? Historiquement, l'industrie de l'aluminium était dominée par un petit groupe de producteurs qui fixait les prix en fonction des coûts de production. Toutefois, en 1978, on a commencé à vendre des contrats sur l'aluminium à la London Metal Exchange, une bourse située à Londres, au Royaume-Uni, spécialisée dans les matières premières. Depuis, d'autres bourses ont emboîté le pas, mais cette dernière demeure une référence pour le marché. Les contrats sur l'aluminium sont des ententes standardisées entre un vendeur et un acheteur concernant la livraison, en un certain lieu, d'une certaine quantité d'un métal d'une certaine qualité. Lorsqu'ils sont faits à l'avance, ces contrats sont dits «futures » et sont vendus à une prime (ou un rabais) près du prix prévu à la date de livraison. Ces contrats peuvent être vendus ou achetés sans que la livraison soit nécessairement réalisée. Comment a évolué le prix de ces contrats ?

Le graphique 1 montre la variation des prix nominal et réel de l'aluminium sur la période allant de 1980 à $2007^{2}$. Le prix illustré est celui du contrat sur l'aluminium de la London Metal Exchange, pureté minimum 99,5\%, prix au comptant, coût, assurance et fret aux ports du Royaume-Uni, en dollars américains par tonne métrique. L'indice de prix utilisé est l'indice de prix des matières premières métalliques du Fonds monétaire international (FMI), qui a 1995 comme année de base, et qui comprend les prix du cuivre, de l'aluminium, du minerai de fer, de l'étain, du nickel, du zinc, du plomb et de l'uranium. On peut voir sur le graphique une tendance à la baisse du prix réel : le prix nominal de l'aluminium a crû moins vite que l'ensemble des prix nominaux des métaux sur la période considérée. En raison de ce qui a été dit précédemment, on pourrait penser que cette tendance, plutôt faible, pourrait ne pas être observée si on choisissait une autre période de temps ou un autre indice de prix. Cependant, nous avons analysé plusieurs sources de données sur les prix, plusieurs indices de prix et calculé les tendances sur plusieurs périodes différentes suffisamment longues, et nous avons toujours observé ce déclin plus ou moins prononcé. Quelles sont les causes de ce phénomène?

La théorie économique fournit plusieurs modèles qui permettent de relier l'évolution des prix à des variables sous-jacentes. Gilbert suggère d'utiliser, pour l'industrie de l'aluminium, le modèle de la concurrence de Cournot, où les producteurs se font concurrence sur la quantité, la qualité et les conditions de livraison plutôt que sur le prix. En pratique, les analystes des marchés des métaux ont tendance à voir l'évolution courante et future en terme d'équilibre entre l'offre et la demande. Cette approche est fondée, mais il faut préciser que les termes « offre » et «demande» doivent être entendus ici dans le sens, respectivement de «quantité offerte » et de «quantité demandée » à un prix donné. À court terme, on considère que la production est limitée par la capacité de production, mais que les producteurs peuvent réagir aux prix du marché en faisant varier leurs stocks ou en n'utilisant, à la limite, pas toutes leurs capacités de production. Enfin, le prix de long terme est plutôt déterminé par les coûts marginaux ${ }^{3}$ et on considère que les capacités de production peuvent varier.

Lorsqu'on veut évoquer le côté de la demande du marché de l'aluminium, on fait souvent référence à la consommation de ce métal. Or, la demande est un concept plutôt difficile à observer dans la pratique. C'est pourquoi on s'intéresse aux utilisations du métal en aval du marché ou encore aux déterminants classiques de la demande, comme le revenu ou la présence de substituts, pour en discuter l'évolution. La croissance économique aura tendance à faire augmenter les quantités demandées pour chaque prix, tandis que la 
présence de substituts rendra la demande d'aluminium plus sensible aux variations de prix.

Gilbert affirme que l'aluminium est consommé de façon assez diffuse dans l'industrie, notamment dans les secteurs de l'électricité, de la construction, du transport et de l'emballage. La substitution se fait par d'autres métaux et d'autres matériaux de construction, comme le cuivre, le plastique ou la fibre de carbone, sans qu'aucun d'eux ne prédomine. La substitution dépend principalement d'avancées technologiques plutôt que des changements dans les prix relatifs. En effet, surtout à court terme, les entreprises demandeuses sont restreintes dans leurs choix de matériaux car elles ne peuvent changer facilement de procédés de production ou de design de produits. Une fois que le marché est passé d'un matériau à un autre, comme cela a été le cas lorsque l'industrie électrique est passée du cuivre à l'aluminium, il faut des mouvements de prix substantiels pour renverser la situation. En conséquence, l'effet des comportements de substitution peut être observé dans la tendance générale de la consommation mais difficilement par le jeu des prix relatifs. Dans le cas de l'aluminium

\section{Prévisions pour les vingt prochaines années}

Avant de donner nos prévisions quant à l'avenir du prix réel de l'aluminium dans les vingt prochaines années, on peut rappeler ce que d'autres ont avancé. Nappi ${ }^{5}$ prévoit « une certaine stabilisation (et même une certaine augmentation) des prix réels au cours de la période 2006-2020». Il invoque plusieurs raisons à cette affirmation. Premièrement, la demande pour l'aluminium devrait croître, selon des prévisions basées sur l'évolution du revenu au niveau mondial, d'environ $4 \%$ par année sur cet horizon. Deuxièmement, «l'industrie internationale de l'aluminium devrait être caractérisée à l'horizon 2020 par un plus haut degré de concentration indus- secondaire, c'est davantage l'importance politique accrue du recyclage que le prix avantageux qui aurait causé un gain en importance de l'aluminium secondaire. Cependant, une fois qu'un système efficace de recyclage est en place, il est généralement moins coûteux d'utiliser le métal secondaire. Certaines productions, comme celle des canettes, se font maintenant principalement à partir de cette source.

Dans les dernières années, la demande mondiale pour l'aluminium a crû avec le revenu mondial. Cela pourrait pousser les prix à la hausse, mais l'offre mondiale d'aluminium a augmenté aussi, poussée par les investissements en capacité et par une augmentation de la productivité. Aussi, selon Figuerola-Ferretti ${ }^{4}$, l'introduction des contrats sur l'aluminium à la bourse a grandement diminué le pouvoir des producteurs sur le prix de l'aluminium et a permis à une plus grande concurrence de s'installer dans l'industrie de l'aluminium. L'effet global est que l'augmentation de l'offre l'emporte sur l'augmentation de la demande et le prix de l'aluminium est entraîné à la baisse, tandis que la quantité du métal échangée sur le marché augmente.

trielle ». Enfin, les coûts de production à long terme devraient augmenter. En effet, la croissance économique fulgurante de pays émergents, comme la Chine, pousse la demande mondiale de nombreux biens et services, dont les intrants dans la production d'aluminium primaire, comme l'énergie, l'alumine et le carbone et par conséquent, leur prix, à la hausse. Aussi, l'économiste affirme qu'il est de plus en plus difficile d'augmenter l'efficacité de la production sans qu'un changement technologique majeur ait lieu. Enfin, une dépréciation du dollar américain, de nature structurelle, devrait «faire augmenter les coûts de production exprimés en dollars ». 
D'un autre côté, le consultant Mark Fraser, du Commodity Research Unit (CRU), affirme, dans une présentation faite devant l'Aluminum Association en 2005, que tandis que la tendance du prix réel de l'aluminium primaire a été à la baisse d'environ $1 \%$ par année pour la période allant de 1960 à 2003 (selon les données utilisées par le CRU et en prenant les prix de l'année 2004 comme base), les vingtcinq prochaines années verront cette tendance à la baisse se poursuivre, mais à un rythme plus faible, soit $0,5 \%$ par année. L'argumentation tient du fait que le prix réel va toujours tendre vers les coûts d'opération de long terme, et que ces coûts vont continuer de diminuer dans le futur. Dans une autre présentation du CRU devant l'Aluminum Association, cette fois donnée en 2006, Mark Barden affirme que la hausse récente du prix nominal est due à une sous-estimation, par les acteurs de l'industrie dans les dernières années, de la demande chinoise. Il y a eu un sous investissement dans le secteur minier en amont, qui a amené une limite à court terme à l'expansion de la capacité de production d'aluminium, dont les projets étaient déjà caractérisés par des coûts du capital plus élevés. Cela, et d'autres facteurs, ont amené des coûts de production croissants dans le secteur de l'aluminium. Cependant, l'auteur affirme qu'à moyen terme, le marché de l'aluminium va revenir plus près de l'équilibre et que les prix vont revenir à un plus bas niveau, notamment grâce à un fort investissement du secteur financier dans toutes les matières premières.

De notre côté, nous affirmons nous aussi que la tendance à la baisse du prix de l'aluminium va se poursuivre sur une longue période. Sur le graphique 1 , la tendance à la baisse n'est que d'environ $0,5 \%$ annuellement sur la période considérée, et nous pensons que ce taux pourrait diminuer légèrement. L'industrie a connu une période de prix nominaux élevés ces dernières années, mais comme l'ont illustré Dupuis et Lefebvre ${ }^{6}$, les prix des métaux sont très cycliques et déjà on voit poindre une correction sur le marché. Les producteurs d'aluminium sont déjà à ajuster leurs capacités de production pour répondre à la demande mondiale croissante. Comme les prix de long terme suivent l'évolution des coûts de production, nous supposons donc que ceux-ci continueront de décroître légèrement. Pourquoi ?

Les éléments qui composent les coûts de production de l'aluminium peuvent être classés en plusieurs catégories : l'énergie, le capital, le travail, les biens et les services intermédiaires. Sur l'ensemble de l'industrie mondiale, les coûts de ces différents éléments peuvent varier considérablement. Pour ce qui est de l'énergie, des sources et des modes de production diversifiés sont utilisés, de sorte qu'une variation dans le prix d'une source ou d'un mode de production peut, à long terme, être compensée par l'utilisation de sources ou de modes relativement moins dispendieux. Enfin, la variation des prix de l'énergie a tendance à diffuser dans le reste de l'économie et à affecter le prix de beaucoup d'autres biens. Il ne faut pas oublier qu'un changement qui affecterait également tous les métaux, ou également l'aluminium et les éléments qui composent l'indice de prix utilisé pour calculer son prix réel, n'aurait que peu ou pas d'impact sur le prix réel du métal gris. Si les coûts reliés à l'énergie augmentaient dans l'avenir, nous pensons que cette augmentation aurait un impact plus modéré sur le prix réel de l'aluminium primaire que ce que laisse croire l'intensité en énergie de cette production.

Les coûts en capital sont sans doute moins variables à travers l'industrie, car ils dépendent des conditions des marchés financiers mondiaux. Cependant, l'intervention des états, qui semble revenir en vogue afin d'offrir de meilleures conditions d'accueil aux entreprises ou même de participer aux nouveaux projets en tant que partenaires, pourrait être une source de diminution des risques financiers et donc des 
coûts du capital dans les prochaines années. Pour ce qui est des coûts du travail, le déplacement d'une partie de la production vers les pays où la main-d'œuvre est abondante et les salaires relativement faibles pourrait contrebalancer une augmentation des ces coûts dans les pays industrialisés. Même le respect de normes environnementales ou sociales plus serrées, croyons-nous, qui pourra entraîner des coûts supplémentaires d'un côté, pourra de l'autre favoriser l'acceptabilité sociale des projets et l'accès aux ressources pour les entreprises. Enfin, les coûts des biens et services intermédiaires sont déterminés sur leurs marchés respectifs. Cependant, il ne semble pas que les ressources en bauxite soient en voie de raréfaction importante dans les prochaines années.

\section{Conclusion}

Dans cet article, nous avons esquissé les tendances qui ont marqué l'industrie mondiale de l'aluminium primaire dans les dernières décennies en nous concentrant sur son prix réel, qui est un indicateur de la performance de l'industrie relativement à une portion plus large, mais reliée, de l'économie. Nous avons affirmé que la tendance à la baisse du prix réel de l'aluminium primaire va se poursuivre, mais de façon modérée. Certes, la demande d'aluminium va sans doute continuer d'augmenter, surtout en raison de la croissance
L'évolution technologique affecte la façon dont les intrants sont combinés pour produire l'aluminium, qui vise une augmentation de la productivité globale de cette activité. Les innovations technologiques des dernières années ont surtout visé à améliorer le procédé existant, par exemple en le rendant plus efficace au niveau énergétique. À moins qu'on ne découvre un tout autre procédé de fabrication, on peut penser qu'il existe des limites à ces améliorations et que les dernières technologies en sont approchées. Cependant, ces technologies récentes ne sont pas encore aussi répandues qu'elles pourraient l'être de par le monde, et les anciennes technologies seront progressivement rempla-cées par cellesci pendant les vingt prochaines années, entraînant des gains moyens de productivité sur l'ensemble de l'industrie.

des économies émergentes, mais nous pensons que les capacités de production vont s'ajuster de façon à répondre à cette demande. Nous croyons également que le marché boursier va continuer d'être un puissant mécanisme favorisant la concurrence et les investissements visant à réduire les coûts de production. Enfin, il faut garder à l'esprit que lorsqu'on essaie de comprendre et de prévoir les phénomènes économiques, on ne doit pas négliger la grande capacité des agents de réagir et de s'adapter à des changements.

\section{Notes et références}

1 GILBERT, Christopher L. "Modelling Market Fundamentals: A Model of the Aluminium Market", Journal of Applied Econometrics, 1995 , vol. 10, pp. 385-410.

2 Il est à noter que même si le contrat sur l'aluminium a été échangé depuis 1978 à la LME, la banque de données historiques du FMI sur les prix des matières premières commence en 1980.

3 Le coût marginal est le coût de la dernière unité produite.

4 FIGUEROLA-FERRETTI, Isabel. «Prices and production cost in aluminium smelting in the short and the long run », Applied Economics, 2005, vol. 37, pp. 917-928.

5 NAPPI, Carmine. «L'industrie internationale de l'aluminium, 1980-2006 : changements structurels et perspectives », Cahiers de l'histoire de l'aluminium, Été 2006, pp. 27-34.

6 DUPUIS, François et Martin LEFEBVRE. «Ralentissement mondial en vue : doit-on s'attendre à une correction marquée des prix des matières premières? », Point de vue économique, Études économiques Desjardins, 17 novembre 2006.

BARDEN, Mark. Aluminium: The Way Ahead, présentation devant l'Aluminum Association, 23 octobre 2006

FRASER, Mark. Aluminium Prices : What should we expect ?, presentation devant l'Aluminun Association, 3-4 octobre 2005.

NAPPI, Carmine. L'aluminium, Paris, Economica, 1994, 118 p. 
Figure 1. Schéma de produits et procédés relatifs à l'aluminium, adapté de Nappi (1994).

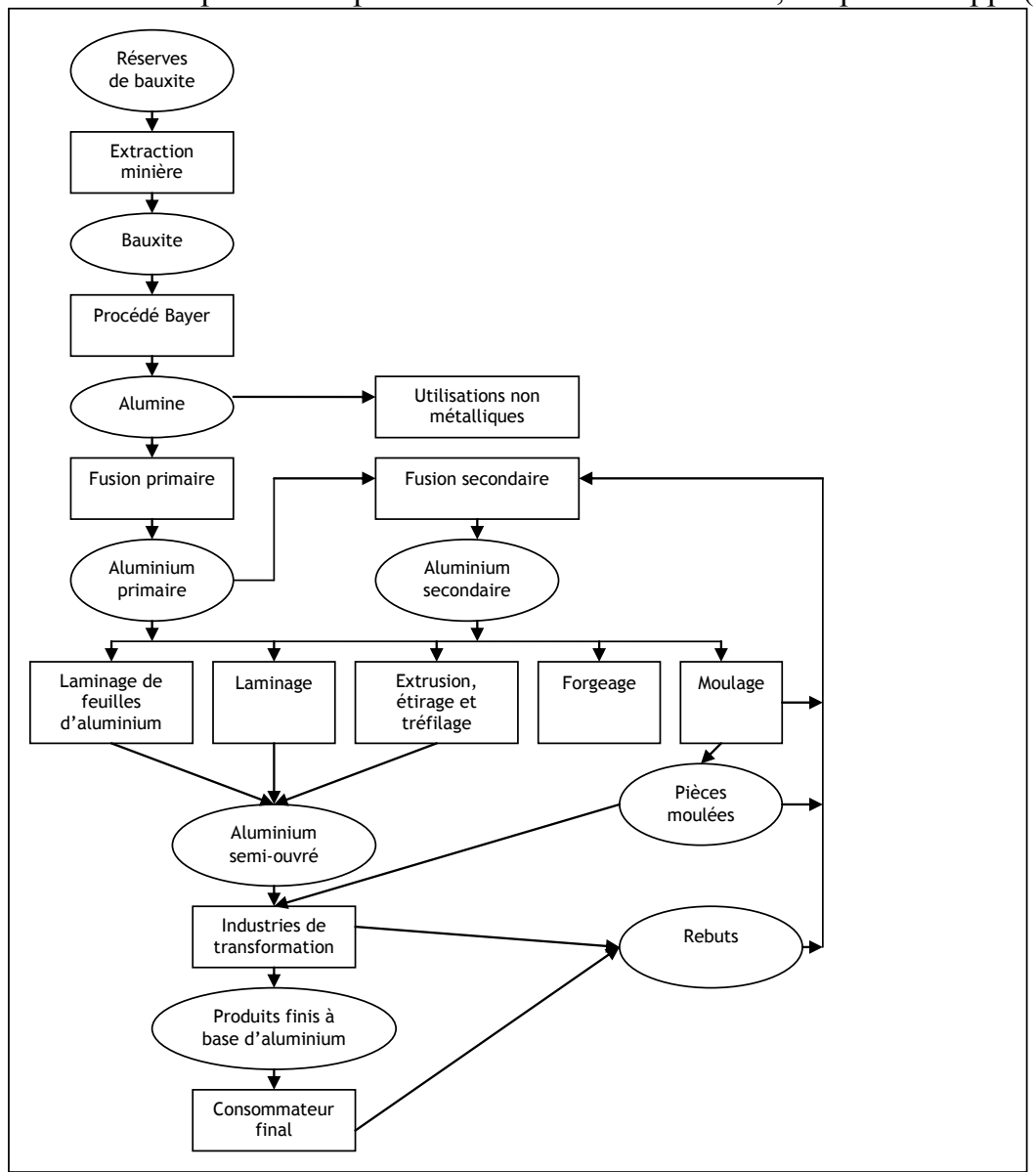

Graphique 1. Source : Fonds Monétaire International, Prix des matières premières ; Traitement : CRDT-UQAC

Prix nominal et réel de l'aluminium primaire (1995=100), London Metal Exchange, données mensuelles, janvier 1980 à septembre 2007

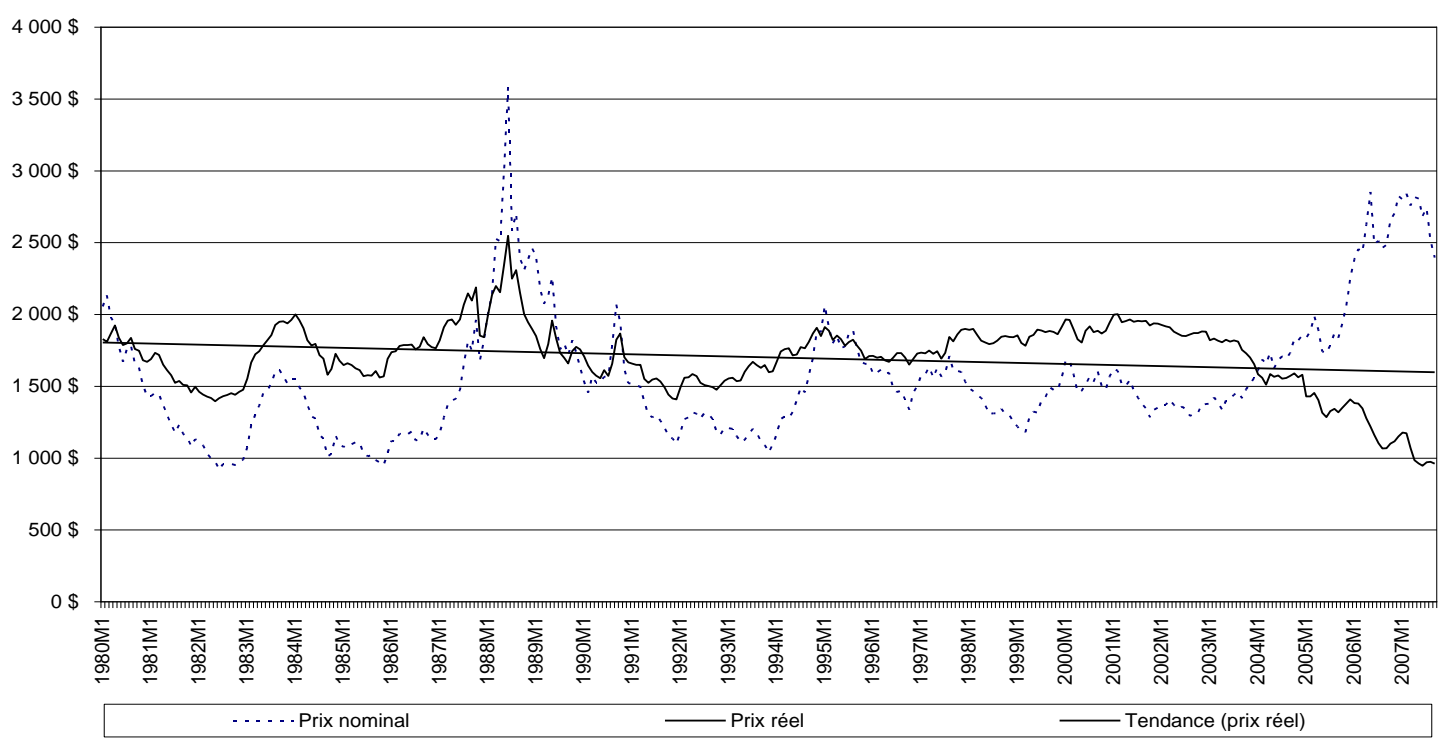

